\title{
Bitcoin in China: Pure Risk Generator or Potential Investment Instrument?
}

\author{
Xiang Li \\ Chinese Academy of Finance and Development, the Central University of Finance and Economics, \\ Beijing, 102206, China \\ hjlx01@126.com
}

Keywords: Bitcoin, Detrended Ratio, Diversification Benefit, Investment Instrument.

\begin{abstract}
In December 2013 the People's Bank of China announced the restriction of Bitcoin from being involved in any services offered by financial institutions in China, fearing that the speculative risks inherent in Bitcoin is too new for Chinese investors and may endanger the national financial system. This article reviews the Bitcoin's evolvement both as currency and as investment asset around the world. Also, the regulatory treatment for Bitcoin in America is discussed. Then I used detrended ratios to compare the volatility of Bitcoin market and China's stock market, concluding that the risks inherent in Bitcoin are not unacceptable for Chinese investors. Moreover, I searched into the relationship between Bitcoin return and some fundamental economic variables, finding that Bitcoin doesn't have enough correlation with the national economic system to depress investors further during an economic downturn and that Bitcoin actually has excellent diversification benefit as portfolio component.
\end{abstract}

\section{Introduction}

Created in 2009 by mysterious Satoshi Nakomoto, Bitcoin has been hastening its popularity around the world ever since. Despite many innovative properties, the inherent conflicts Bitcoin possess against the government's monetary policy, which will be rendered useless if Bitcoin ever becomes legitimate currency, are the reason why it has not received much political interests in developing countries, especially those holding the belief that government's interference in the market is a necessity. For instance, in December 2013, the People's Bank of China announced the restriction of transactional use of Bitcoin, which denied Bitcoin as currency [1]. However, the government did admit Bitcoin's status as virtual commodity and as long as the financial risk is fully exposed to the public, Bitcoin could be traded with investors bearing the entire losses should any occur. On the other hand, the government still firmly segregated the Bitcoin from financial system, prohibiting any kind of Bitcoin-involved financial services. This article intends to review the development history of Bitcoin in the United States, which, as will be revealed, inevitably leads to its role as a strategic component in the investment portfolio. Then the econometrics techniques are employed to understand the speculative nature of Bitcoin in China and estimate the diversification benefit Bitcoin may have to enhance the efficiency of investors' portfolio.

\section{Literature review of Bitcoin's evolvement}

\subsection{The monetary value of Bitcoin}

Naturally, tremendous amounts of the scholarly interests attracted into this area have centered on the viability of Bitcoin to serve as a currency. First of all, Noah Smith has discussed the replacement of fiat money by cryptocurrency such as Bitcoin would be significantly favored in countries where hyperinflation is present [2]. Moreover, Beat Weber tried to assess Bitcoin's potential to create input and output legitimacy as a replacement for current ones [3]. Also, T. Swamy and Shukla have researched into pros and cons of Bitcoins to become the legitimate currency in India under the background of booming e-commerce industry and ongoing technological advances in every field [4]. Moreover, Aaron Pelc took a practical perspective of Bitcoin, looking into the move of Hastings-on- 
Hudson, New York-based Straub Auto Repairs to accept Bitcoin as a form of payment [5]. Similarly, Ivaschenko found that accepting Bitcoin as the payment actually helped some small and medium enterprises in Ukraine improve their investment attractiveness [6].

However, disagreeing voices can also be heard regarding the monetary value of Bitcoin. They mainly targeted the extremely volatile characteristics of Bitcoin, arguing that it's even more volatile than some emerging market currencies [7]. Furthermore, despite the proponents' continuous insisting that Blockchain could prevent the fraudulent activities from happening, in 2014 Mt.Gox organization, the largest Bitcoin exchange in the world, carried out the largest fraud, sending Bitcoin price all the way down from $\$ 750$ to $\$ 225$ [8]. The blow to the optimistic supporters for Bitcoins in investment as well as academic community was almost irreversible.

\subsection{The regulatory treatment for Bitcoin in the U.S.}

Although the monetary future of Bitcoin still remains to be seen, because of the surging trading volume of it nowadays, the regulatory treatments for Bitcoin have developed in a corresponding manner. In the incipient stage, for five years Bitcoin did not have a clear taxation status in the U.S. with disagreeing views centering whether Bitcoin should be treated as property or as currency. Not until March 26 ${ }^{\text {th }}, 2014$ did Internal Revenue Service (IRS) release its IRS Notice 2014-21 announcing that Bitcoin and other crypto-currencies do not have legal tender status and will be treated as property for U.S. federal tax purposes, which leads to difficulties with reporting for many traders [9]. Since Bitcoin also has some currency characteristics, which make it extremely difficult to calculate the basis value and tax the correspondent capital gains [10]. Tax evasion became almost inevitable. On May $7^{\text {th }}, 2014$, however, Stockman the Representative introduced the Virtual Currency Tax Reform Act proposing shifting tax status of virtual currencies from property to foreign currency. Requiring only normal income taxes and the sales tax whenever Bitcoin changes hand, this Act is obviously the more proper way to tax Bitcoins. It never passed the Congress, though.

\section{Empirical study of Bitcoin market in China}

As mentioned above, although the monetary value of Bitcoin has been denied in China, the government still allows Bitcoin trading as long as the investors themselves bear all potential risks. However, Bitcoin-related investment services are strictly forbidden in any financial institutions, which means that Bitcoin market is basically an institution-free market in China. While all the other Bitcoin exchanges daily trading volume remains approximately 10,000 coins, BTC China, the largest exchange in China has a trading volume amounting to 3 million coins. Gigantic amounts of capital and the individual investors playing a leading role are two features not so unfamiliar for Chinese investors. The other one starts much earlier and has earned itself a reputation for extremely fierce volatility. Now let's compare the risks possessed between stock market and Bitcoin market in China and find out whether the alleged "speculative risks" presumed by our central bank are anything new for national financial system.

\subsection{Data description}

Bitcoin price data are downloaded from bicoincharts.com as daily prices denoted in CNY from June 2011 to March 2017. Fundamental economic and financial data are collected from Wind Database, including Consumer Price Index, industrial production, fixed asset investment, CSI 300 Index, 10-year national bond. The monthly frequency is employed throughout the research.

\subsection{Stock market and Bitcoin market}

Naturally the most intuitive indicator to compare risks inherent in two markets is the volatility. Since we may clearly observe an increasing trend in both time series during the time period in discourse, the detrending work is needed for direct comparison purpose. The exponential trend is found through the following minimization:

$$
\operatorname{Min} \sum_{t=1}^{n}\left(X_{t}-Y_{t}\right)^{2}
$$


Where $X_{t}$ stands for the actual value while $Y_{t}=a e^{r t}+b$. The appropriate values for $a, r$ and $b$ are calculated to minimize the sum of squares. Table 1 presents the coefficients estimated for two markets respectively.

Table 1. Estimated coefficients for the exponential trend

\begin{tabular}{|c|c|c|}
\hline \multicolumn{1}{|c|}{ Estimated coefficient } & Bitcoin & CSI 300 Index \\
\hline $\mathrm{a}$ & 529.3133 & 785.0754 \\
\hline $\mathrm{r}$ & 0.03529 & 0.0247 \\
\hline $\mathrm{b}$ & -259.964 & 1610.304 \\
\hline
\end{tabular}

Figure 1 and Figure 2 report the exponential trend for Bitcoin value and CSI 300 Index value respectively.

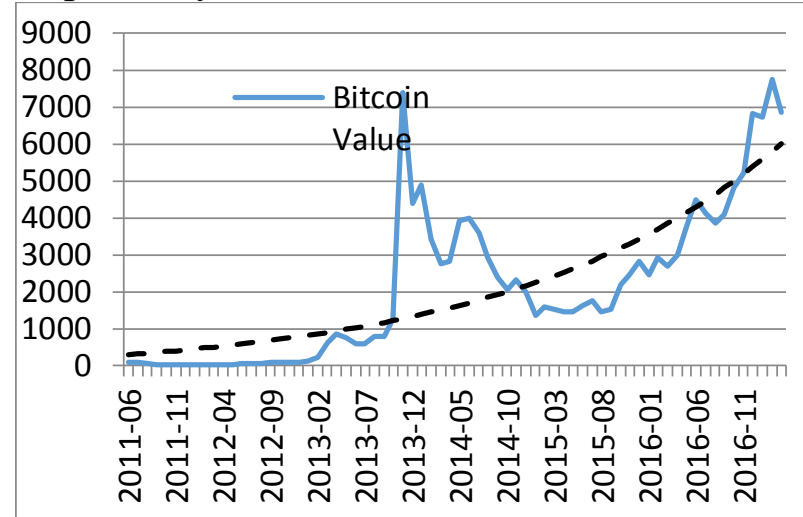

Fig. 1. Exponential

Trend for Bitcoin Value

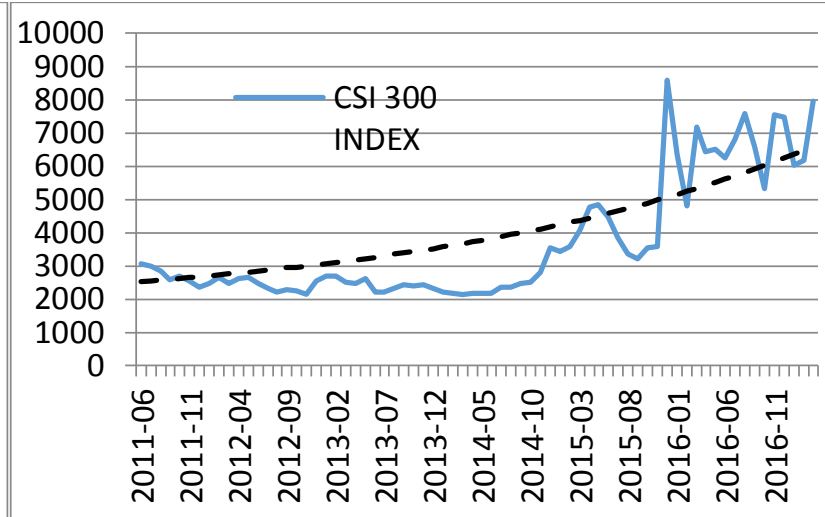

Fig. 2. Exponential Trend for CSI 300 Index

Subsequently in an effort to rule out the interference of dimension, we calculated the detrended ratio of the two different markets. The results are shown in Figure 3 and Figure 4.

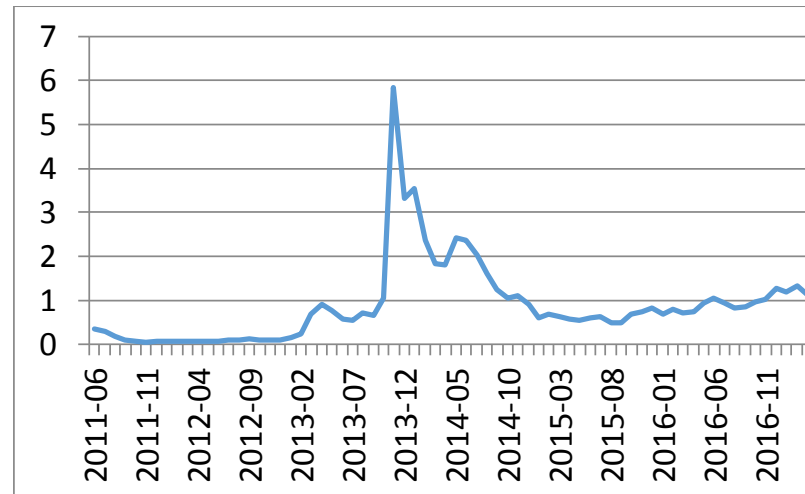

Fig. 3. Detrended ratio of Bitcoin Value

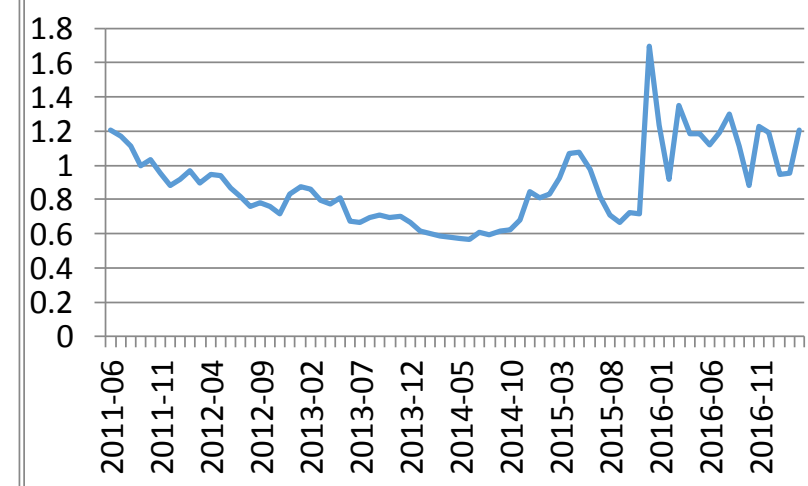

Fig. 4 Detrended ratio of CSI 300

Also, the statistical measures of detrended ratios may be compared in Table 2.The standard deviation for Bitcoin market is 0.9152, while the SD for CSI 300 Index is 0.1540. At first glance we may conclude that the Bitcoin market is about 6 times as volatile as the stock market during the time period in discourse and thus indicate that the government is right to make up relevant regulations preventing Bitcoin from playing a role in the investment portfolio. However, further reflection almost immediately detects the flaws in this argument. First of all, the same ratio in the United States, that is to say the SD of S\&P 500 Index relative to the American Bitcoin Value, is as large as 26 times. Taking this into consideration, either the Bitcoin market in China is not as risky as expected or the stock market in China is already very volatile, which makes the risks inherent in Bitcoin not anything new to the financial system of China. Secondly, as compared to the stock market in the United States, stock market in China actually has imposed price variation range of $20 \%$, not to mention the circuitbreaker mechanism which further reduces the variation the market would have. Last but not least, we notice the positive skewness and kurtosis for CSI Index market, which indicates that the extreme values are just as likely to occur in the stock market. 
Table 2. Statistical measures of detrended ratios

\begin{tabular}{|c|c|c|}
\hline \multicolumn{1}{|c|}{ Statistical measure } & Bitcoin & CSI 300 Index \\
\hline SD & 0.9152 & 0.1540 \\
\hline Skewness & 2.1010 & 1.1207 \\
\hline Kurtosis & 8.4884 & 3.9972 \\
\hline
\end{tabular}

\subsection{The diversification benefit of Bitcoin}

Now that Bitcoin proves to be not as risky as the authority indicated, I want to extend the topic further and find some roles Bitcoin may play in an investment portfolio. Since the original suspicion held by the regulator is that Bitcoin may introduce huge amounts of risks into the national financial system and possibly help trigger some economic crisis. With regard to this concern I conduct a regression analysis using fundamental economic and financial variables. Newey-West's heteroscedasticity and autocorrelation consistent covariance (HAC) estimators are employed in the regression:

$$
\mathrm{R}_{\mathrm{t}}=\beta_{0}+\beta_{1} \Delta \mathrm{cpi} \mathrm{i}_{\mathrm{t}}+\beta_{2} \Delta \mathrm{ip}_{\mathrm{t}}+\beta_{3} \Delta \mathrm{fai}_{\mathrm{t}}+\beta_{4} \Delta \mathrm{NB}_{\mathrm{t}}+\beta_{5} \Delta \mathrm{csi} 300_{\mathrm{t}}+\beta_{6} \Delta \operatorname{spread}_{\mathrm{t}}+\varepsilon_{\mathrm{t}}
$$

Where $\mathrm{R}_{\mathrm{t}}$ is the monthly change in Bitcoin price, $\Delta \mathrm{cpi}_{\mathrm{t}}$ is the monthly change in the Consumer Price Index, $\Delta \mathrm{ip}_{\mathrm{t}}$ is the monthly change in industrial production, $\Delta \mathrm{fai}_{\mathrm{t}}$ is the monthly change in fixed asset investment, $\Delta \mathrm{NB}_{\mathrm{t}}$ is the monthly change in 10-year national bond yield, $\Delta \mathrm{csi} 300_{\mathrm{t}}$ is the monthly change in CSI 300 Index and $\Delta$ spread $_{\mathrm{t}}$ is the monthly change in the spread between daily high and low Bitcoin prices. Except $\Delta \operatorname{spread}_{t}$, all the other variables are external factors. This regression analysis may help us determine whether Bitcoin has the diversification benefit especially in the face of national economic downturn.

\begin{tabular}{|c|c|c|}
\hline \multicolumn{2}{|c|}{ Table 3. Regression results } \\
\hline \\
Variable & $\begin{array}{c}\text { Estimated } \\
\text { coefficient }\end{array}$ & t-ratio \\
\hline Constant & -0.4613 & -1.1283 \\
\hline Monthly change in consumer price index & -0.1693 & -0.9948 \\
\hline Monthly change in industrial production & 0.00302 & 0.0683 \\
Monthly change in fixed asset investment & 0.4241 & 1.6046 \\
Monthly change in 10-year national bond & -0.0515 & -0.3820 \\
Monthly change in CSI 300 Index & 0.3742 & 0.5416 \\
Monthly change in the spread between daily high & & \\
and low prices & $0.0345^{* *}$ & 2.1751 \\
Adjusted $\mathbf{R}^{2}$ & 0.2304 & \\
\hline
\end{tabular}

Table 3 reports regression results. We're surprised to find that not a single fundamental economic or financial variable is statistically significant. The only significant coefficient belongs to the internal factor, the spread between daily high and low prices. This suggests that the Bitcoin market is actually internally driven and the authority is right about Bitcoin's speculative nature. However, totally uncorrelated with the conventional investment assets and those economic variables, Bitcoin will certainly possess the benefits of diversification. When national economic system is going through a downturn, the Bitcoin won't antagonize it. When stock market and bond market deteriorates, Bitcoin price won't be influenced. As we should intuitively know, as a completely new tradable asset, Bitcoin has to have great efficiency in promoting the risk-return trade-off indicator of a portfolio. Analogous to emerging market currencies, which have been shown to be great instruments for balancing general investment portfolios, Bitcoin should have the similar attractiveness. In the United States many of the major investment companies offer emerging market ETFs. And John Carrick has conducted researches in which he introduces Bitcoin into the basic currency basket of these ETFs. As a result, the Sharpe Ratio and Sortino Ratio for these baskets are increased dramatically. And when the occupancy of Bitcoin shifts from equally weighted to $10 \%$ of the basket, the ratios are improved even further. In China, the Bitcoin is as uncorrelated with macroeconomic variables as in America and has relatively lower volatility, which makes it more likely that Bitcoin has the power to promote the risk- 
return trade off in many financial institution's portfolios, so long as the government removes those bans.

\section{Conclusion}

This article researches into the correlation between Bitcoin expected return and many fundamental economic and financial variables. It turns out that Bitcoin market is strictly internally driven and won't aggravate any investment portfolio should economic downturn or financial market deterioration happens. Also, this reveals that Bitcoin should have excellent potential for diversification concerns. As discussed in the end of proceeding section, many major financial institutions in America have employed emerging market currencies as instruments to balance general investment portfolios. And researchers in America have found that including Bitcoin in the basic basket promotes its function efficiency even further. I firmly believe the same applies to China if these practices are allowed. Hopefully in the near future Chinese government will discover the benefits Bitcoin has as an investment asset and remove the bans over financial institutions to include this virtual currency in their portfolio. Then, already with the largest amount of Bitcoin transactions going on daily, China will play an even bigger role in Bitcoin's resurrection.

\section{References}

[1]. "Notice for preventing the risks of Bitcoin" [2013] \#289, issued by People's Bank of China, Ministry of Industry and Information, China Banking Regulatory Commission, China Securities Regulatory Commission, China Insurance Regulatory Commission.

[2]. Noah Smith. Will Bitcoin Ever Grow Up to be Money? Fort worth Business Press. 3/14/2016, Vol. 28 Issue 10, p38-38. 1p.

[3]. Beat Weber. Bitcoin and the Legitimacy Crisis of Money. Cambridge Journal of Economics. Jan2016, Vol. 40 Issue 1, p17-41. 25p.

[4]. Swamy T., Pramanshu Shukla, Shreyas Ganesan Iyer, Rishi Gupta. Review Paper on Emergence of Bitcoin in India, its Technological Aspects and Legal Implications. Global Management Review May2016, Vol. 10 Issue 3, p55-60. 6p.

[5]. Aaron Pelc. Bitcoin Buy Auto Repairs. Westchester County Business Journal. 6/9/2014, Vol. 50 Issue 23, p2-2. 1p.

[6]. Ivaschenko A. I. Using Cryptocurrency in The Activities of Ukrainian Small and Medium Enterprises in order to Improve Their Investment Attractiveness. Problems of Economy. 2016, Issue 3, p267-273. 7p.

[7]. Jon Carrick. Bitcoin as a Complement to Emerging Market Currencies. Emerging Markets Finance \& Trade. 2016, Vol. 52 Issue 10, p2321-2334. 14p.

[8]. Kristoufek L. What are The Main Drivers of The Bitcoin Price? Evidence from Wavelet Coherence Analysis. 2015 Plos One 10(4): e0123923. Doi: 10.1371/journal. Pone.0123923

[9]. Elizabeth E. Lambert. The Internal Revenue Service and Bitcoin: A Taxable Relationship. Virginia Tax Review. Summer2015, Vol. 35 Issue 1, p88-115. 28p.

[10]. Roman José Andre. Bitcoin: Assessing the Tax Implications Associated with the IRS's Notice Deeming Virtual Currencies Property. Review of Banking \& Financial Law. Spring2015, Vol. 34 Issue 2, p451-457. 7p. 